\title{
A Generalized Window Approach for Designing Transmultiplexers
}

\author{
Pilar Martín-Martín, Member, IEEE, Robert Bregović, Member, IEEE, Alfonso Martín-Marcos, \\ Fernando Cruz-Roldán, Senior Member, IEEE, and Tapio Saramäki, Fellow, IEEE
}

\begin{abstract}
This paper proposes a computational, very efficient, approach for designing a novel family of $M$-channel maximally decimated nearly perfect-reconstruction cosine-modulated transmultiplexers. This approach is referred to as the generalized windowing method for transmultiplexers because after knowing the transmission channel a proper weighted sum of the inter-channel and inter-symbol interferences can be properly taken into account in the optimization of the window function, unlike in other existing windowing techniques. The proposed approach has also the following two advantages. First, independent of the number of subchannels and the common order of the subchannel filters, the number of unknowns is only four. Second, the overall optimization procedure is made considerably fast by estimating the above-mentioned sum in terms of two novel measures, namely, the signal to inter-symbol and the signal to inter-channel interferences, which are very easy to evaluate. Furthermore, when the transmission channel is not considered in the design, a table is provided, which contains the parameters for designing the prototype filter directly by using the windowing method without any time-consuming optimization. When comparing the resulting transmultiplexers with the corresponding perfect-reconstruction designs (the same number of subchannels and same prototype filter order), the levels of interferences are practically the same. However, when the system is affected by a strong narrowband interference, the proposed transmultiplexers outperform their PR counterparts. Design examples are included illustrating the efficiency of the proposed design approach over other existing techniques based on the use of the windowing method.
\end{abstract}

Index Terms-Filterbanks, linear phase, nearly-perfect reconstruction, optimization, transmultiplexers.

\section{INTRODUCTION}

C URRENTLY, the most widely used multicarrier transmission (MCT) technology in wireline and wireless transmissions are the discrete multitone transmission (DMT) [1] and the orthogonal frequency-division multiplexing (OFDM) [2]. In both these systems, the modulation and demodulation are performed digitally using the inverse discrete Fourier transform

Manuscript received February 1, 2007; revised August 20, 2007 and December 3, 2007. First published March 07, 2008; current version published October 29, 2008. This work was supported by the Academy of Finland under Project 213462 [Finnish Centre of Excellence Program (2006-2011)]. This paper was recommended by Associate Editor B. C. Levy.

P. Martín-Martín and F. Cruz-Roldán are with the Department of Teoria de la Señal y Comunicaciones, University of Alcal á, Alcalá de Henares, 28871 Madrid, Spain (e-mail: p.martin@uah.es; fernando.cruz@uah.es).

A. Martín Marcos is with the Department of Ingenieria Audiovisual y Comunicaciones, Universidad Politécnica, 28031 Madrid, Spain (e-mail: amartin@diac.upm.es).

R. Bregović and T. Saramäki are with the Department of Signal Processing, Tampere University of Technology, FIN-33101 Tampere, Finland (e-mail: bregovic@cs.tut.fi; ts@cs.tut.fi).

Digital Object Identifier 10.1109/TCSI.2008.920095
(IDFT) and the discrete Fourier transform (DFT), respectively. The attractive features of these two systems are a low design complexity and an easy equalization process accomplished with the aid of a cyclic prefix. Inserting such a cyclic prefix in the system, however, considerably reduces the payload of the transmission. The second drawback of these two systems is that a narrowband interference could degrade several subchannels and make them unusable due to the fact that the attenuation of the first sidelobe in the subchannel filters is approximately only $13 \mathrm{~dB}$

The significant spectral overlap between subchannel filters in DFT-based MCT systems can be avoided by using alternative filterbanks-based techniques, known as the filterbanks-based multicarrier transmission systems (FB-MCTs), such as the overlapped DMT [3], [4], filtered multitone transmission (FMT) [5], or the discrete wavelet multitone transmission (DWMT) [6]. These FB-MCT systems use finite-impulse response (FIR) filters that are longer than the rectangular filters used in DFT-based MCT systems, thereby resulting in better subchannel spectral containment. Moreover, there are efficient techniques for implementing FB-MCTs [7]. For this purpose, modulated transmultiplexers (MTs), such as compleX-modulated transmultiplexers (CXMTs), cosine-modulated transmultiplexers (CMTs) and sine-modulation transmultiplexers (SMTs) are good candidates since in these systems the synthesis and analysis filters are generated in efficient manners based on the use of one or two prototype filters. However, their main problem is the equalization process due to the use of long filters [8]. In order to solve this problem, the MTs (FB-MCTs) must have a large number of subchannels. In this case the frequency response of the transmission channel in each subchannel can be considered flat and the equalization can be performed in a very simple way, that is, by using a single tap frequency-domain equalizer per subchannel. However, such a requirement makes the design of the prototype filters for generating MTs very difficult because the number of coefficients involved in the optimization process tends to be very large.

This paper concentrates on $M$-channel CMTs with subchannel filters of order $N=2 K M-1$, where $K$ is an integer, that is called the overlapping factor [9] and $M$ is the number of subchannels. Fixing the subchannel filters order in the above manner is motivated by the fact that when designing perfect-reconstruction (PR) systems, only for these orders there are no severe restrictions on any value of the impulse-response coefficients of the prototype filter (see, e.g., [10]). It is worth mentioning that, due to the relation among MTs, the approach proposed in this paper can be applied equally well to the design of prototype filters for SMTs and CXMTs [10]-[13]. 


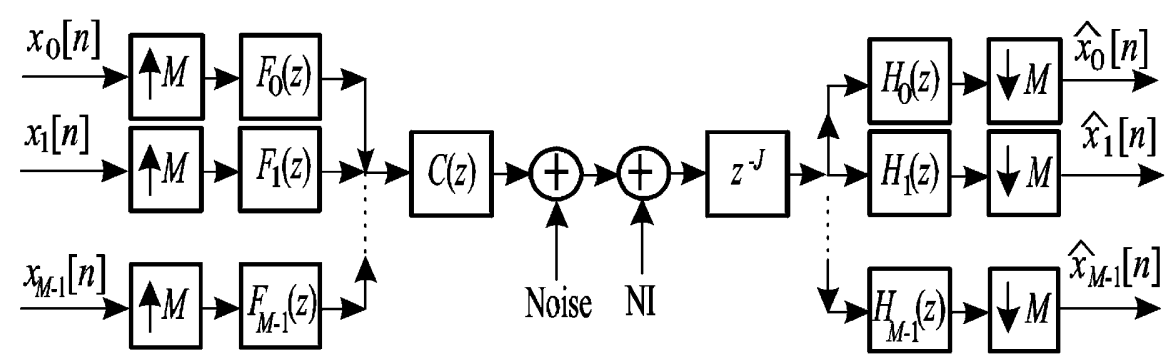

Fig. 1. $M$-channel maximally decimated transmultiplexer over a transmission channel.

Additionally, due to the duality between multirate filterbanks and transmultiplexers, the approach proposed in this paper can also be applied to the design of filterbanks.

This paper focuses on nearly-perfect reconstruction (NPR) CMTs due to the fact that in most applications, the transmission channel itself introduces a considerable distortion. NPR CMTs relax the PR condition by allowing small inter-channel interference (ICI) and inter-symbol interference (ISI). Allowing these small interferences is beneficial since it enables one to achieve better stopband performances compared with the PR case.

The main contributions of this paper can be summarized as follows.

1) When taking into account the transmission channel, new analytical expressions are proposed for evaluating the signal-to-ICI ratio (SICI), signal-to-ISI ratio (SISI), and signal-to-overall-interference ratio (SI).

2) For optimization purposes a new objective function is provided, which properly controls the trade-off between interferences for a given transmission channel.

3) A computationally efficient approach is proposed for designing prototype filters for a novel family of NPR CMTs. The optimization problem has always four unknowns, independently of the order of the subchannel filters. This enables one to design extremely selective transmultiplexers with a reduced design complexity.

4) Finally, when the transmission channel is not considered in the design approach, a table is provided containing the parameters that are required for the design of a prototype filter by exploiting the windowing method (WM) [14] without any time-consuming optimization. By using this table, systems with very long subchannel filters and good interference levels can be designed without any optimization.

This paper is organized as follows: The NPR CMTs are briefly reviewed in Section II. Section III gives closed-form expressions for measuring the ICI and ISI when taking into account the transmission channel. The design problem is stated in Section IV together with an efficient algorithm for solving this problem. Section V provides some design simulations in order to clarify when and/or where the proposed approach is a suitable choice. Finally, in Section VI some concluding remarks are given.

\section{Cosine-Modulated TRANSmultipleXers}

An $M$-channel transmultiplexer is an $M$-channel filterbank, where the synthesis block (transmitter) precedes the analysis block (receiver) in order to form a system with $M$ inputs and $M$ outputs, as depicted in Fig. 1 [15]. At the transmitter, the $M$ input signals are, first, interpolated by a factor of $M$, secondly, filtered by the $M$ synthesis filters $F_{k}(z)$ for $k=0,1, \ldots, M-1$, and, finally, added to form a single signal for transmission over a given transmission channel $C(z)$. At the receiver, the signal is then split back into $M$-channels for generating the desired $M$ outputs, that is, the outputs $\widehat{x}_{k}[n]$ for $k=0,1, \ldots, M-1$ that carry as well as possible the information included in the corresponding input signals $x_{k}[n]$. This is performed by, first, filtering the signal by the $M$ analysis filters $H_{k}(z)$ for $k=$ $0,1, \ldots, M-1$ and, then, decimating the output signals of the analysis filters by a factor of $M$.

According to discussion in [16]-[18], an $M$-channel NPR filterbank will not lead to an $M$-channel NPR transmultiplexer unless the filterbank introduces a delay of $D=r M$ samples with $r$ being an integer. In this case, the corresponding transmultiplexer introduces an integer value delay of $D_{T}=(r M) / M=r$ samples between the corresponding output and input signals. Therefore, in order to ensure the NPR property, in most cases a delay term $z^{-J}$ should be inserted in the transmultiplexer before the demultiplexer block, as shown in Fig. 1 [19]. For instance, if the order of the subchannel filters is $N=2 K M-1$, then a delay term $z^{-1}$ has to be used in order to make $D=r M$ with $r=2 K$.

In CMTs, all the analysis and synthesis filters can be easily generated by modulating an $N$ th-order lowpass linear-phase prototype filter with impulse response $p[n]$ as follows [20]:

$$
\begin{aligned}
& h_{k}[n]=2 p[n] \cos \left[\left(k+\frac{1}{2}\right) \times \frac{\pi}{M}\left(n-\frac{N}{2}\right)+(-1)^{k} \frac{\pi}{4}\right] \\
& f_{k}[n]=2 p[n] \cos \left[\left(k+\frac{1}{2}\right) \times \frac{\pi}{M}\left(n-\frac{N}{2}\right)-(-1)^{k} \frac{\pi}{4}\right]
\end{aligned}
$$

for $k=0,1, \ldots, M-1$.

In the $z$-domain, the relation between the output in the $k$ th subchannel $\widehat{x}_{k}[n]$ and all the inputs $x_{l}[n]$ for $l=0,1, \ldots, M-1$ can be written as

$$
\widehat{X}_{k}\left(z^{M}\right)=\frac{1}{M} \sum_{l=0}^{M-1} T_{k l}\left(z^{M}\right) X_{l}\left(z^{M}\right)
$$

where $T_{k l}\left(z^{M}\right)$ describes the transfer function between the $l$ th input and $k$ th output (at the transmission channel sampling rate) and is given by

$$
T_{k l}\left(z^{M}\right)=\sum_{i=0}^{M-1} F_{l}\left(z W^{i}\right) C\left(z W^{i}\right) H_{k}\left(z W^{i}\right)
$$


for $k, l=0,1, \ldots, M-1$ and $W^{i}=e^{-j(2 \pi i) /(M)} \cdot{ }^{1}$ For $l \neq k$, this function describes the crosstalk function between the $k$ th and the $l$ th subchannels. For $l=k$, in turn, it describes the direct transfer function in the $k$ th subchannel.

\section{General Measures for the Signal-TO-ICI AND SIGNAL TO ISI}

The ICI in each subchannel is the undesirable effect of the remaining $M-1$ inputs on that subchannel. The ICI in the $k$ th subchannel can be conveniently measured as follows [21]:

$$
E_{\mathrm{ICI}}^{(k)}=\frac{1}{\pi} \int_{0}^{\pi}\left(\sum_{l=0, l \neq k}^{M-1}\left|T_{k l}\left(e^{j \omega}\right)\right|^{2}\right) d \omega
$$

where $T_{k l}\left(e^{j \omega}\right)$ can be obtained from (3). On the other hand, the ISI in that subchannel is the undesirable effect due to the fact that the transmitted sample does not occur at the output at the right time instant. The ISI in the $k$ th subchannel can be measured as follows [21]:

$$
E_{\mathrm{ISI}}^{(k)}=\frac{1}{\pi} \int_{0}^{\pi}\left\{\left\|T_{k k}\right\|_{1}-\left|T_{k k}\left(e^{j \omega}\right)\right|\right\}^{2} d \omega
$$

where

$$
\left\|T_{k k}\right\|_{1}=\frac{1}{\pi} \int_{0}^{\pi}\left|T_{k k}\left(e^{j \omega}\right)\right| d \omega
$$

represents the $L_{1}$ norm of the frequency response $T_{k k}\left(e^{j \omega}\right)$.

The previous two measures are good enough for measuring the ICI and ISI in the $k$ th subchannel if the transmission channel is not included in the computations. However, when including the transmission channel, the ICI and ISI cannot be evaluated without taking into consideration how the transmission channel is affecting the direct transfer function of that subchannel. Therefore, in this paper, two new measures, namely, the signal to inter-channel interference (SICI) and the signal to inter-symbol interference (SISI) are proposed.

The $\mathrm{SICI}_{k}$ measures the ratio between the signal and the crosstalk interference levels in the $k$ th subchannel. It can be evaluated by using the following formula:

$$
\mathrm{SICI}_{k}=\frac{E_{k}}{E_{\mathrm{ICI}}^{(k)}}
$$

where

$$
E_{k}=\frac{1}{\pi} \int_{0}^{\pi}\left(\left|T_{k k}\left(e^{j \omega}\right)\right|^{2}\right) d \omega
$$

is the energy of the $k$ th direct transfer function and $E_{\mathrm{ICI}}^{(k)}$, as given by (4), is the energy of the ICI in the same subchannel.

The SISI $k$, in turn, measures the ratio between the signal and the ISI levels in the $k$ th subchannel. It is evaluated by

$$
\mathrm{SISI}_{k}=\frac{E_{k}}{E_{\text {ISI }}^{(k)}}
$$

where $E_{k}$ and $E_{\mathrm{ISI}}^{(k)}$ are given by (8) and (5), respectively.

\footnotetext{
${ }^{1} j$ stands for the imaginary part of a complex number, as opposed to $i$, which is used for indexing.
}

Furthermore, a global measure for the signal-to-overall interference ratio (SI) in the $k$ th subchannel, denoted by $\mathrm{SI}_{k}$, can be defined as

$$
\mathrm{SI}_{k}=\frac{E_{k}}{E_{\mathrm{ICI}}^{(k)}+E_{\mathrm{ISI}}^{(k)}}=\frac{1}{\left(\mathrm{SICI}_{k}\right)^{-1}+\left(\mathrm{SISI}_{k}\right)^{-1}}
$$

It measures the ratio between the energy of the direct transfer function in the $k$ th subchannel and the energy of the overall interference in that subchannel.

In order to compare the proposed approach with other design approaches, a measure being independent of the design procedure has to be used. A good candidate for that purpose is the signal-to-noise ratio (SNR). The SNR in the $k$ th subchannel $\left(\mathrm{SNR}_{k}\right)$ is defined by

$$
\mathrm{SNR}_{k}=\frac{\sigma_{x_{k}}^{2}}{\sigma_{e_{k}}^{2}}=\frac{\sum_{n}\left(x_{k}[n]\right)^{2}}{\sum_{n}\left(e_{k}[n]\right)^{2}}
$$

where

$$
e_{k}[n]=\widehat{x}_{k}\left[n-D_{T}\right]-x_{k}[n]
$$

Here, $D_{T}$ is the overall system delay and $x_{k}[n]$ and $\widehat{x}_{k}[n]$ are the input and the output signals in the $k$ th subchannel, respectively. The SNR of the overall transmultiplexer is defined as the average of the $\mathrm{SNR}_{k}$ in all subchannels, that is,

$$
\mathrm{SNR}=\frac{1}{M} \sum_{k=0}^{M-1} \mathrm{SNR}_{k} .
$$

In order to show the relationships of the new measures proposed in this paper, that is, the $\mathrm{SICI}_{k}, \mathrm{SISI}_{k}$ and $\mathrm{SI}_{k}$, with the $\mathrm{SNR}_{k}$, an analysis was performed as follows. First, a 32-channel PR CMT was designed by using the approach proposed in [10] in the $K=3$ case $^{2}$. Secondly, the transmission channel was selected to have the frequency-domain characteristics as shown in Fig. 2. Fig. 3(a)-(c) displays the $\mathrm{SICI}_{k}, \mathrm{SISI}_{k}, \mathrm{SI}_{k}$ and $\mathrm{SNR}_{k}$ for $k=0,1, \ldots, M-1$. By comparing these figures, the following two observations can be made: First, the performance of the $\mathrm{SI}_{k}$ follows rather closely the worse one among those of the $\mathrm{SICI}_{k}$ and $\mathrm{SISI}_{k}$ and second, the proposed measure $\mathrm{SI}_{k}$ provides a very good information about the $\mathrm{SNR}_{k}$. Therefore, by using only the measures $\mathrm{SICI}_{k}$ and $\mathrm{SISI}_{k}$ for $k=0,1, \ldots, M-$ 1 enables one to design CMTs that have a good performance over the transmission channel also in terms of the values of $\mathrm{SNR}_{k}$ for $k=0,1, \ldots, M-1$. Moreover, these measures can be evaluated very fast and, most importantly, in straightforward manners. These facts emphasizes the usefulness of the new measures of "goodness" proposed in this section.

\section{Proposed Generalized Windowing ApPRoACH}

Up to now, several approaches have been proposed for designing prototype filters for CMTs, for instance [22] and [23]. In this section, a computationally efficient approach for designing linear-phase $N$ th-order FIR prototype filters for $M$-channel

\footnotetext{
${ }^{2}$ Recall from Section I that this contribution concentrates only on prototype filters of order $N=2 K M-1$, where $K$, called overlapping factor, is an integer.
} 


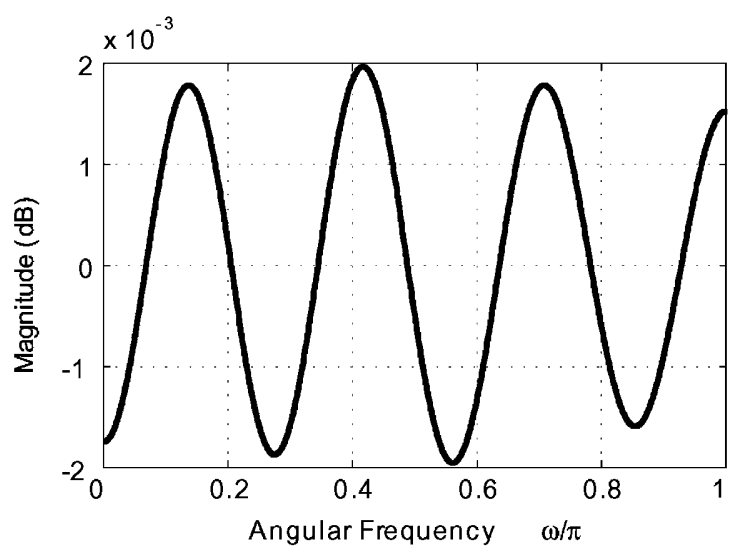

(a)

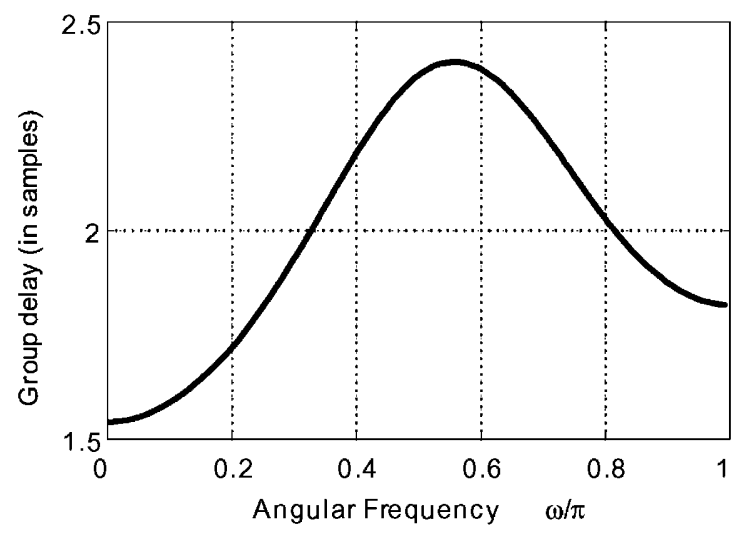

(b)

Fig. 2. Nonlinear frequency-selective transmission channel under consideration. (a) Magnitude response. (b) Group delay.

maximally decimated NPR CMTs is proposed, called Generalized Windowing Method for Transmultiplexers (GWMT). The key idea in this approach is to design the prototype filter in such a manner that it provides CMTs for a given transmission channel with a properly selected trade-off between the ICI and ISI by using a very simple and consequently very fast design algorithm. The corresponding optimization problem and an efficient unconstrained optimization algorithm for solving it are given. When the transmission channel is not considered in the design approach, a table with the parameters that are required for the design of a prototype filter without time-consuming optimization is also provided.

\section{A. Windowing Method for FIR Filter Design}

The proposed GWMT approach is based on the Windowing Method (WM). In the WM, the values of the impulse-response coefficients $p[n]$ of a causal $N$ th-order linear-phase FIR filter are simply generated for $0 \leq n \leq N$ as

$$
p[n]=w[n] h_{c}[n]
$$

where $h_{c}[n]$ is the impulse response of a causal ideal lowpass filter given as

$$
h_{c}[n]= \begin{cases}\frac{\sin \left[\omega_{c}(n-N / 2)\right]}{\pi(n-N / 2)}, & \text { for } 0 \leq n \leq N \\ 0, & \text { otherwise }\end{cases}
$$

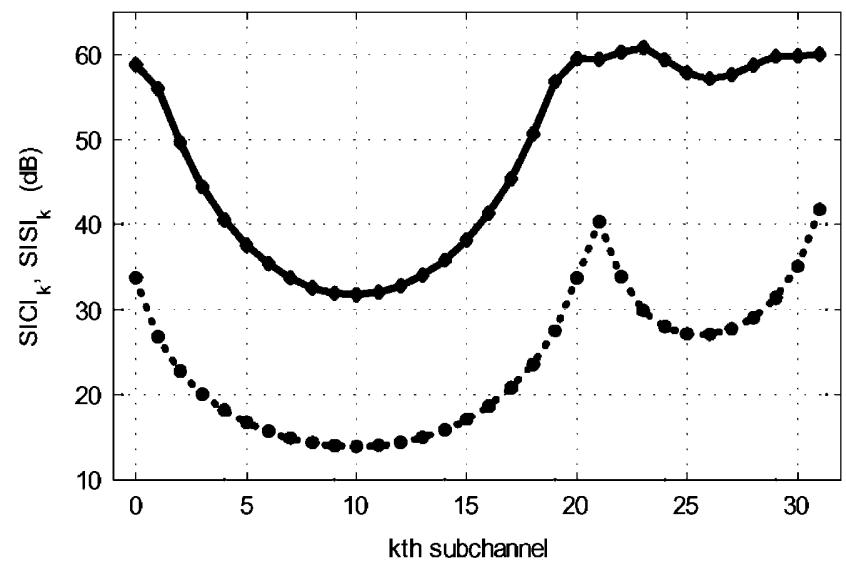

(a)

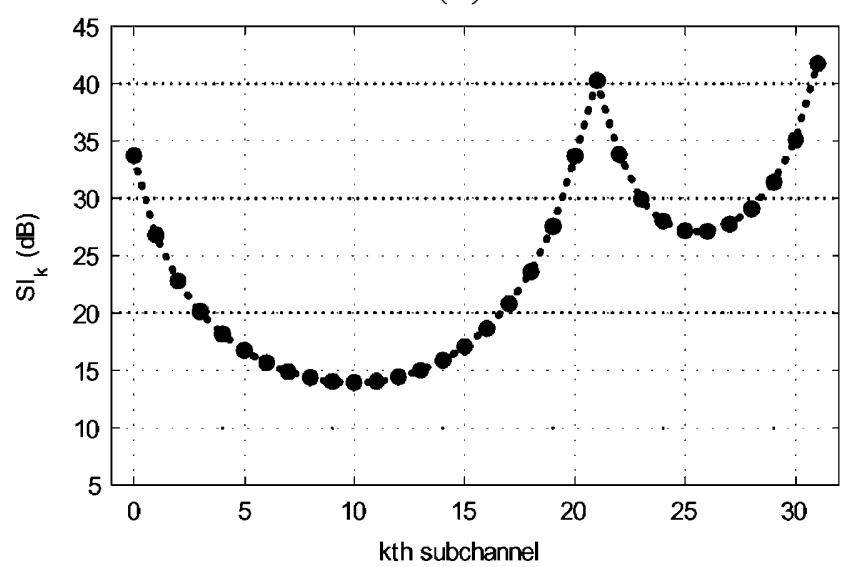

(b)

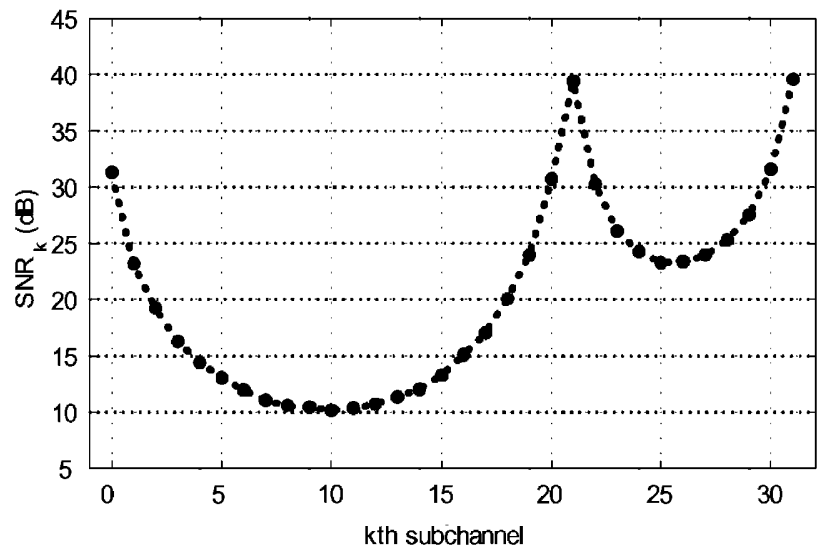

(c)

Fig. 3. Performances in the $k$ th subchannel for $k=0,1, \ldots, 31$ of a 32-channel PR CMT in the $K=3$ case over the nonlinear frequency-selective transmission channel shown in Fig. 2. (a) $\mathrm{SICI}_{k}$ (dotted line) and $\mathrm{SISI}_{k}$ (solid line). (b) $\mathrm{SI}_{k}$. (c) $\mathrm{SNR}_{k}$.

with $\omega_{c}$ being the cut-off frequency of the causal ideal lowpass filter and $w[n]$ is the window function satisfying $w[N-n]=$ $w[n]$.

The window function under consideration in this paper is the following four-term generalized cosine window function:

$$
w[n]=\sum_{i=0}^{3}(-1)^{i} A_{i} \cos \left(\frac{2 \pi \mathrm{in}}{N}\right)
$$




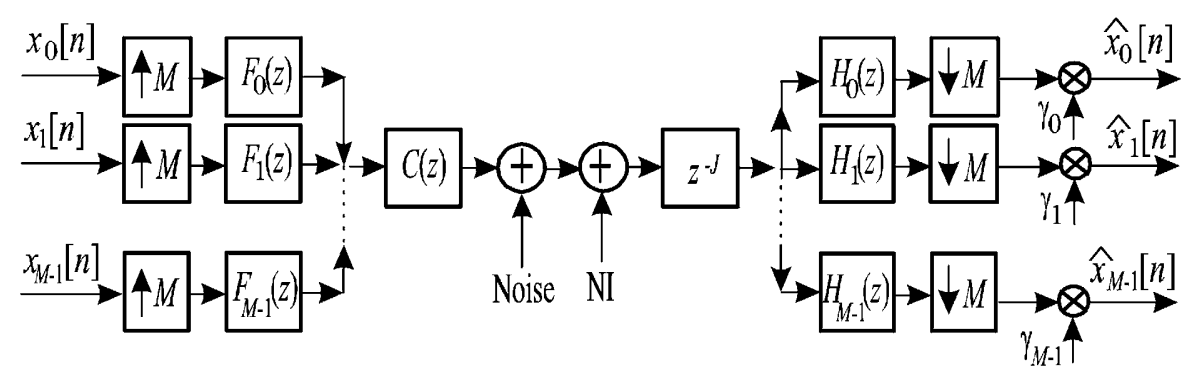

Fig. 4. GWMT-based $M$-channel transmultiplexer configuration.

for $n=0,1, \ldots, N$. The $A_{i}$ values are the weights of the terms for $i=0,1,2,3$. Without loss of generality, this window function is normalized, according to [14], [24]-[26], as

$$
\sum_{i=0}^{3} A_{i}=1
$$

\section{B. Statement of the Optimization Problem}

In the proposed general problem formulation, the prototype filter order $N$, the number of subchannels $M$, and the compromise factor $\alpha$ between the ISI and ICI are fixed before the optimization procedure is started. The weights $A_{i}$ in the generalized cosine window function and the cut-off frequency of the ideal lowpass filter $\omega_{c}$ are adjusted by minimizing the following objective function:

$$
\phi(\mathbf{x})=-\frac{1}{M} \sum_{k=0}^{M-1} \frac{E_{k}(\mathbf{x})}{\alpha E_{\mathrm{ICI}}^{(k)}(\mathbf{x})+(1-\alpha) E_{\mathrm{ISI}}^{(k)}(\mathbf{x})}
$$

where $E_{k}(\mathbf{x}), E_{\mathrm{ICI}}^{(k)}(\mathbf{x})$, and $E_{\mathrm{ISI}}^{(k)}(\mathbf{x})$ are given by (8), (4), and (5), respectively. The factor $0 \leq \alpha \leq 1$ controls the weights of the ICI and ISI. When the requirements of the application are known beforehand, the value of $\alpha$ can be selected such that it appropriately emphasizes the importance of the interference that is more crucial for the application at hand. For instance, when emphasizing both interferences in the same way, $\alpha=0.5$ is a good selection.

The adjustable parameter vector $\mathbf{x}$ contains only four adjustable terms, independently of the subchannel filter order and the number of subchannels, that is,

$$
\mathbf{x}=\left[A_{0}, A_{1}, A_{2}, \omega_{c}\right]
$$

After finding the weights $A_{0}, A_{1}$ and $A_{2}$, the remaining weight $A_{3}$ is determined according to the condition of (17).

The analysis and synthesis filters are generated from the prototype filter by using the cosine-modulation scheme, as given by (1). Moreover, in the overall structure for implementing GWMT-based CMTs, as shown in Fig. 4, additional correction factors $\gamma_{k}$ that make the average of $\left|T_{k k}\left(e^{j \omega}\right)\right|$ equal to unity are included. The desired factors are given by

$$
\gamma_{k}=\frac{\max \left\{\left|T_{k k}\left(e^{j \omega}\right)\right|\right\}+\min \left\{\left|T_{k k}\left(e^{j \omega}\right)\right|\right\}}{2} .
$$

\section{Efficient Algorithm for Solving the Optimization Problem}

There exist several approaches for designing prototype filters for CMTs that are based on constrained or unconstrained optimization problems. In most of these problems, the unknowns are the impulse-response coefficients of the prototype filter. Moreover, the objective function and the constraints are highly nonlinear with respect to the unknowns. Therefore, very complicated nonlinear optimization algorithms have to be used.

Search methods that use only function evaluations are the most suitable for problems that are highly nonlinear or have a large number of discontinuities. Gradient methods are generally more efficient when the function to be minimized is continuous in its first derivative. Methods using higher order derivatives, such as Newton's method, are only suitable when the second-order information can be easily evaluated because calculating the second-order information, using numerical differentiation, is computationally expensive.

The proposed optimization problem is hard to solve analytically and it is difficult to guarantee the arrival at the global optimal solution. However, it has turned out that a local optimal solution can be obtained numerically by using the Nelder-Mead simplex minimization algorithm [27]. This optimization algorithm is very efficient from the time consuming and proper final solution points of view. The Nelder-Mead Simplex minimization algorithm uses only function values, that is, it is a direct search method that does not use numerical or analytic gradients [28]. For this purpose, the function fminsearch from the optimization toolbox provided by MathWorks, Inc. [29] is used.

Additionally, for a given optimization problem, it is important to find a good starting point. In most cases this is not trivial. Fortunately, for solving the above proposed optimization problem, based on the experimental data, a good common starting point of the adjustable parameter vector that can be used for all the designs, i.e., independently of the number and order of the subchannels filters, is

$$
\mathrm{x}=[0.42,0.5,0.08, \pi / 2 M]
$$

In this case, the four-term generalized cosine window function is initialized at the Blackman window function parameters and the cut-off frequency of the ideal lowpass filter is located at $\pi / 2 M$, that is, the theoretical position of the $\omega_{3 \mathrm{~dB}}$ of the prototype filter for designing CMTs. ${ }^{3}$

${ }^{3} \omega_{3 \mathrm{~dB}}$ is the frequency at which the magnitude response of the prototype filter $\left|P\left(e^{j \omega}\right)\right|$ achieves the value of $1 / \sqrt{2}$. 
TABLE I

OPTIMIZEI WEIGTHS IN THE GENERALIZEI) COSINE WINIDOW FUNCTIONS ANI Cut-off Frequency of the IdEAl LowPass Filter in the $2 \leq K \leq 8$ CASES FOR $\alpha=0,0.5$, and 1

\begin{tabular}{|c|c|c|c|c|c|}
\hline & $K$ & $A_{0}$ & $A_{1}$ & $A_{2}$ & $\omega_{c} \cdot M$ \\
\hline \multirow{8}{*}{$\alpha=0$} & 2 & 0.3232 & 0.5818 & 0.0784 & 1.7232 \\
\hline & 3 & 0.4224 & 0.4199 & 0.0877 & 1.9200 \\
\hline & 4 & 0.4108 & 0.4961 & 0.0872 & 1.9848 \\
\hline & 5 & 0.5002 & 0.5330 & 0.0321 & 1.8800 \\
\hline & 6 & 0.3841 & 0.5000 & 0.1124 & 1.8688 \\
\hline & 7 & 0.4804 & 0.4838 & 0.0341 & 1.7744 \\
\hline & 8 & 0.3850 & 0.5000 & 0.1113 & 1.7928 \\
\hline & $K$ & $A_{0}$ & $A_{1}$ & $A_{2}$ & $\omega_{c} \cdot M$ \\
\hline \multirow{8}{*}{$\alpha=0.5$} & 2 & 0.5353 & 0.4595 & 0.0524 & 2.0944 \\
\hline & 3 & 0.5703 & 0.4508 & -0.0258 & 1.9898 \\
\hline & 4 & 0.4859 & 0.4863 & 0.0281 & 1.9288 \\
\hline & 5 & 0.5060 & 0.5088 & 0.0231 & 1.8632 \\
\hline & 6 & 0.3733 & 0.4981 & 0.1234 & 1.8776 \\
\hline & 7 & 0.4746 & 0.4862 & 0.0378 & 1.7768 \\
\hline & 8 & 0.3851 & 0.5000 & 0.1113 & 1.7928 \\
\hline & $K$ & $A_{0}$ & $A_{1}$ & $A_{2}$ & $\omega_{c} \cdot M$ \\
\hline \multirow{7}{*}{$\alpha=1$} & 2 & 0.8390 & 0.1601 & 0.0116 & 2.2368 \\
\hline & 3 & 0.4389 & 0.4893 & 0.0728 & 0.9704 \\
\hline & 4 & 0.4058 & 0.4971 & 0.0969 & 1.2912 \\
\hline & 5 & 0.3655 & 0.4920 & 0.1347 & 1.1512 \\
\hline & 6 & 0.3271 & 0.4755 & 0.1728 & 1.2088 \\
\hline & 7 & 0.3347 & 0.4791 & 0.1653 & 1.2920 \\
\hline & 8 & 0.3243 & 0.4744 & 0.1757 & 1.4984 \\
\hline
\end{tabular}

\section{Tabulated Parameters}

When the proposed GWMT approach is used for designing prototype filters for CMTs without including the transmission channel in the proposed design approach, it is straightforward to first provide a table with the required parameters and, then, to design, based on these parameters, the prototype filters using only the WM, without involving any time-consuming optimization. This simplified procedure is motivated by the following experimentally observed facts.

1) The optimized values of the weights $A_{i}$ for $i=0,1,2,3$ in the generalized cosine window function, as given by (16), depend only on the integer parameter $K=(N+1) / 2 M$. Table I shows their values in the $2 \leq K \leq 8$ cases for $\alpha=0, \alpha=0.5$, and $\alpha=1$. The attractive property of the optimized window functions is that their shapes for the given values of $\alpha$ and $K$ remain practically the same as $M$ is varied.

2) The optimized value of the cut-off frequency of the ideal lowpass filter $\omega_{c}$ does not only depend on the number of subchannels $M$, but also on the parameter $K$. This is illustrated in Table I that provides the optimized values for $\omega_{c}$.

3) Fig. 5 shows the $3 \mathrm{~dB}$ cut-off frequency $\omega_{3 \mathrm{~dB}}$ of the prototype filters and its theoretical position $(\pi / 2 M)$ when designing 8-channel CMTs in the $2 \leq K \leq 8$ cases for the

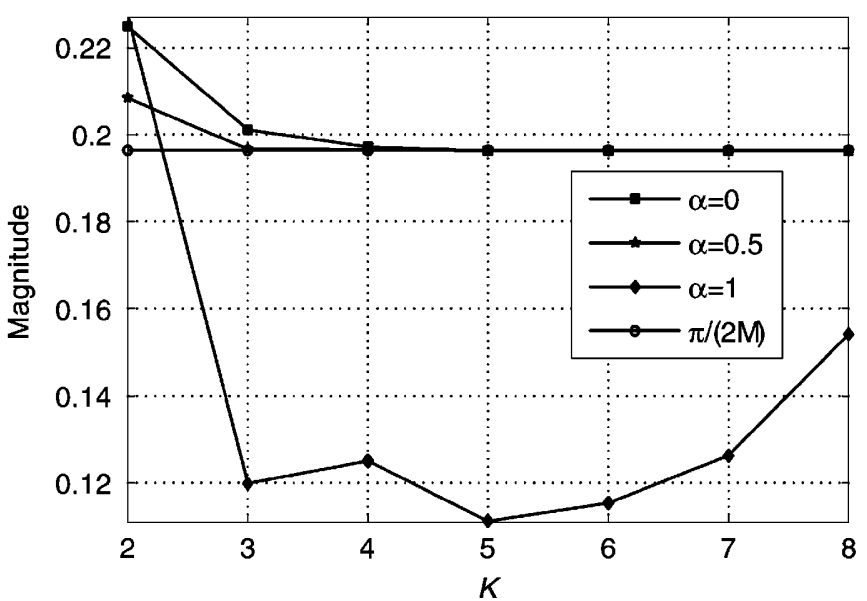

Fig. 5. Values of $\omega_{3 \mathrm{~dB}}$ and $\pi / 2 M$ when designing GWMT-based 8-channel CMTs for $\alpha=0,0.5$ and 1 (see footnote 3 , for the definition of $\omega_{3 \mathrm{~dB}}$ ).

TABLE II

PeRformance of GWMT-BASED CMTS IN THE $2 \leq K \leq 8$ CASFS FOR $\alpha=00.5$, and 1

\begin{tabular}{|c|c|c|c|c|}
\hline & $K$ & $S I C I$ & $S I S I$ & $S I$ \\
\hline \multirow{8}{*}{$\alpha=0$} & 2 & 19.68 & 320.4 & 19.68 \\
\hline & 3 & 26.40 & 316.2 & 26.40 \\
\hline & 4 & 50.08 & 313.8 & 50.08 \\
\hline & 5 & 65.25 & 88.89 & 65.23 \\
\hline & 6 & 85.65 & 108.37 & 85.63 \\
\hline & 7 & 75.38 & 74.52 & 71.92 \\
\hline & 8 & 88.80 & 84.37 & 83.04 \\
\hline & $K$ & SICI & $S I S I$ & $S I$ \\
\hline \multirow{8}{*}{$\alpha=0.5$} & 2 & 68.49 & 111.9 & 68.49 \\
\hline & 3 & 54.95 & 59.16 & 53.56 \\
\hline & 4 & 66.61 & 70.95 & 65.25 \\
\hline & 5 & 71.85 & 82.91 & 71.52 \\
\hline & 6 & 87.13 & 95.06 & 86.48 \\
\hline & 7 & 76.99 & 72.25 & 70.99 \\
\hline & 8 & 88.82 & 84.37 & 83.04 \\
\hline & $K$ & $S I C I$ & $S I S I$ & $S I$ \\
\hline \multirow{7}{*}{$\alpha=1$} & 2 & 90.95 & 21.65 & 21.65 \\
\hline & 3 & 83.49 & 8.39 & 8.39 \\
\hline & 4 & 107.7 & 8.61 & 8.61 \\
\hline & 5 & 126.1 & 6.10 & 6.10 \\
\hline & 6 & 143.4 & 6.18 & 6.18 \\
\hline & 7 & 151.5 & 6.06 & 6.06 \\
\hline & 8 & 155.1 & 9.38 & 9.38 \\
\hline
\end{tabular}

same three $\alpha$ values as considered earlier. It can be observed that the optimized $\omega_{3 \mathrm{~dB}}$ has the closest value to $\pi / 2 M$ for $\alpha=0.5$, that is, when both interferences are emphasized in the same way. However, if the application needs CMTs with very good ICI levels, $\alpha=1$ has to be chosen. In this case, $\omega_{3 \mathrm{~dB}}$ has values quite different from $\pi / 2 M$. 


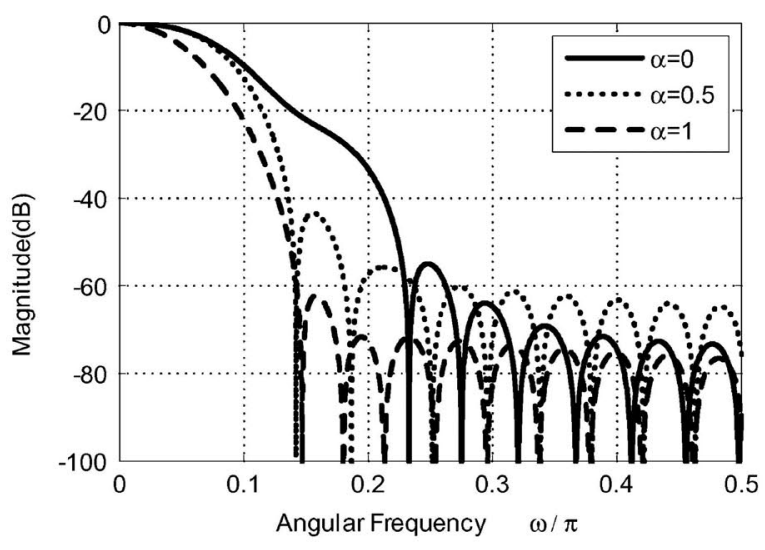

Fig. 6. Magnitude responses of the optimized prototype filters for generating GWMT-based 8-channel CMTs in the $K=3$ case for $\alpha=0, \alpha=0.5$, and $\alpha=1$.

4) The interference level is practically independent of the number of subchannels. It depends mainly on the parameter $K$. Therefore, the results presented here are valid for any number of subchannels as long as $N=2 K M-1$. Table II shows the SICI, SISI, and SI for the GWMT-based CMTs in the $2 \leq K \leq 8$ cases for $\alpha=0, \alpha=0.5$, and $\alpha=1^{4}$. It can be observed that values of the interferences depend on the selected $\alpha$ value. Selecting $\alpha=0.5$ provides the CMTs with the best SI performance. Smaller (larger) values of $\alpha$ improve the SISI (SICI) by emphasizing in the objective function that interference. It is worth noticing that in the $\alpha=0$ case, the algorithm minimizes the objective function, as given by (18), as much is possible. However, there is always a limit for the SICI that depends on the $K$ value, that is, on the stopband attenuation attained by the corresponding order of the prototype filter.

In order to illustrate the magnitude responses of the optimized prototype filters, Fig. 6 shows the responses of the optimized prototype filters for GWMT-based 8-channel CMTs in the $K=$ 3 case for $\alpha=0, \alpha=0.5$, and $\alpha=1$.

\section{Design Simulations}

In this section, in order to illustrate the usefulness of the design approach proposed in this paper, the performances of various 32-channel NPR CMTs are compared in the $K=3$ and $K=7$ cases. The following design approaches are included in the comparison.

1) The proposed GWMT approach for $\alpha=0.5$. The weights of the generalized cosine window function and the values of $\omega_{c}$ are given in Table I.

2) The windowing method for designing filterbanks (WMFB) introduced in [30] using the Blackman window.

3) The Kaiser window approach (KWA) proposed in [23]. For comparison purposes, the minimum stopband attenuation $A_{s}$ is chosen to be equal to the $A_{s}$ of the proposed GWMT-

${ }^{4}$ When the transmission channel is not considered in the design approach, the $\mathrm{SICI}_{k}, \mathrm{SISI}_{k}$ and $\mathrm{SI}_{k}$ do not change from subchannel to subchannel. In this case, the same nomenclature is used but without the index $k$.

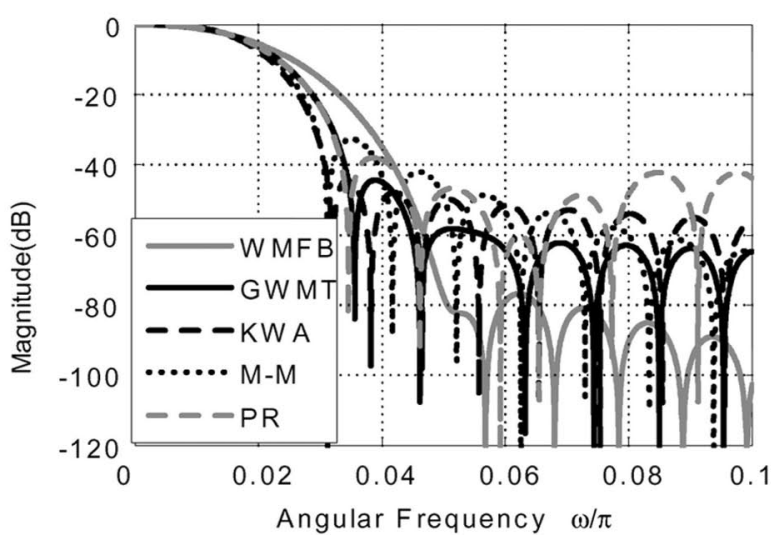

(a)

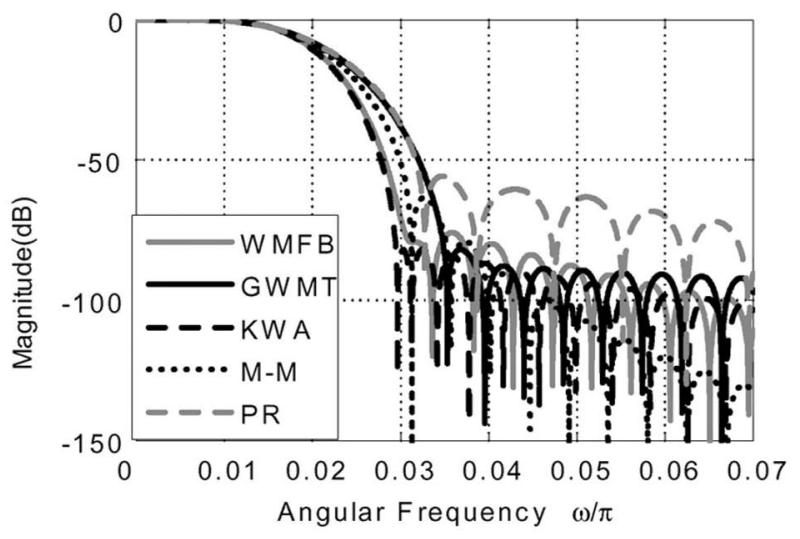

(b)

Fig. 7. Magnitude responses of the prototype filters for generating 32-channel CMTs. (a) $K=3$. (b) $K=7$.

based NPR CMT for the same number of subchannels and order of the filters.

4) The Mirabbasi-Martin approach (M-M) proposed in [26]. The impulse response coefficients of the prototype filters in the M-M approach depend on the overlapping factor $K$ and are given in [26, Table I].

5) A PR CMT with the prototype filter designed in the leastmean-square sense [10].

Fig. 7 shows the resulting magnitude responses of the prototype filters for these five design approaches. Tables III and IV report the values of the SICI, SISI, and SI for the designs under consideration in the $K=3$ and $K=7$ cases, respectively. In order to show the performances of these systems from another point of view, the tables provide also the SNR values as defined by (13).

Tables III and IV show that the GWMT approach provides among the four NPR approaches under consideration the CMTs with the highest values of SI for both overlapping factors ( $K=$ 3 and $K=7$ ). This is a direct consequence of the fact that this measure is optimized in the GWMT approach. Furthermore, the GWMT-based CMTs also provide the highest values for the SNR. These results show that optimizing the interference levels in the proposed manner results in a maximized value for the SNR.

In order to show the performances of the above-mentioned five designs in some practical environments, the next three subsections compare the different CMTs in cases, where the noise, 
TABLE III

PERHORMANCES OH 32-ChANNEL CMTS UNDER CONSIIDERATION IN THE $K=3$ CASE

\begin{tabular}{c|ccccc}
\hline \hline & GWMT & $\begin{array}{c}\text { WMFB } \\
{[30]}\end{array}$ & $\begin{array}{c}\text { KWA } \\
{[23]}\end{array}$ & $\begin{array}{c}\text { M-M } \\
{[26]}\end{array}$ & $\begin{array}{c}\text { PR } \\
{[10]}\end{array}$ \\
\hline SICI $(\mathrm{dB})$ & 54.95 & 35.77 & 49.50 & 43.87 & 286.54 \\
$S I S I(\mathrm{~dB})$ & 59.16 & 37.42 & 32.21 & 54.26 & 291.13 \\
$S I(\mathrm{~dB})$ & 53.56 & 33.50 & 32.13 & 43.49 & 283.52 \\
$S N R(\mathrm{~dB})$ & 54.43 & 33.35 & 31.03 & 43.15 & 280.45 \\
\hline \hline
\end{tabular}

TABLE IV

Performances of 32-ChanNel CMTS Under Consideration IN THE $K=7 \mathrm{CASE}$

\begin{tabular}{c|ccccc}
\hline & GWMT & $\begin{array}{c}\text { WMFB } \\
{[30]}\end{array}$ & $\begin{array}{c}\text { KWA } \\
{[23]}\end{array}$ & $\begin{array}{c}\text { M-M } \\
{[26]}\end{array}$ & $\begin{array}{c}\text { PR } \\
{[10]}\end{array}$ \\
\hline$S I C I(\mathrm{~dB})$ & 76.99 & 92.64 & 98.50 & 98.87 & 278.93 \\
$S I S I(\mathrm{~dB})$ & 72.25 & 65.28 & 62.72 & 66.82 & 289.83 \\
$S I(\mathrm{~dB})$ & 70.99 & 65.27 & 62.75 & 66.82 & 278.59 \\
$S N R(\mathrm{~dB})$ & 69.69 & 65.08 & 61.83 & 66.62 & 275.09 \\
\hline \hline
\end{tabular}

narrowband interference, and transmission channel affect the overall systems.

\section{A. Effects of an Additive White Gaussian Noise}

The noise used in this simulation is an additive white Gaussian noise (AWGN) with zero mean. The transmitted signal to AWGN noise ratio is defined by

$$
\operatorname{SNR}_{\mathrm{TC}}=\frac{\operatorname{PSD}\{y[n]\}}{\operatorname{PSD}\{e[n]\}}
$$

where $\operatorname{PSD}\{y[n]\}$ is the power spectral density (PSD) of the transmitted signal and PSD $\{e[n]\}$ is the PSD of the AWGN. The maximum value of the $\operatorname{PSD}\{y[n]\}$ in the overall frequency band for a given $\operatorname{PSD}\{e[n]\}$ in the transmission channels in use is specified by standards. For the simulations, the $\operatorname{PSD}\{y[n]\}$ is fixed to be $-60 \mathrm{dBm} / \mathrm{Hz}$ and the frequency band in use is $15 \mathrm{MHz}$, which corresponds to the very high Discrete Subscriber Line (VDSL) transmission considered in [31]. Fig. 8 shows the resulting values of the SNR as functions of $\mathrm{SNR}_{\mathrm{TC}}$ in the $K=3$ case. Based on this figure, the following two observations can be made. First, for low values of $\mathrm{SNR}_{\mathrm{TC}}$, that is, $\mathrm{SNR}_{\mathrm{TC}}<25 \mathrm{~dB}$, all CMTs have practically the same performance due to the high level of noise. Secondly, for higher values of $\mathrm{SNR}_{\mathrm{TC}}$, the proposed GWMT-based CMT has the performance closest to that of the PR CMT among the remaining four NPR designs.

\section{B. Effects of a Narrowband Interference}

In this simulation, a narrowband interference is added to the systems analyzed in the previous section. This narrowband interference being as a result of the use, for instance, an amateur radio, is assumed to consist of a very strong sinusoidal component, for which the interference frequency is located at $7.27 \mathrm{MHz}$. This is a proper selection in order to place the frequency of this interference in the very close vicinity of the

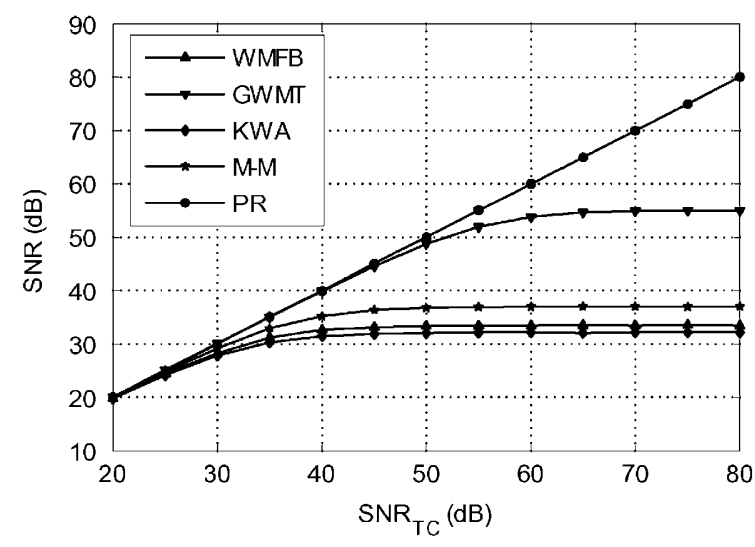

Fig. 8. Values of the SNR for the 32-channel CMTs under consideration in the $K=3$ case when AWGN is added to the systems.

center of the 15th subchannel of a 32-channel CMT in the $15-\mathrm{MHz}$ transmission band case.

As it was observed in previous section when not taking into account the effect of a narrowband interference(see Fig. 8), the really noticeable differences in the performances among the five CMTs under consideration in this contribution occurred for the values of $\mathrm{SNR}_{\mathrm{TC}}$ higher than $25 \mathrm{~dB}$. Therefore, for the following simulations, $\operatorname{PSD}\{e[n]\}=-115 \mathrm{dBm} / \mathrm{Hz}$, that is, $\mathrm{SNR}_{\mathrm{TC}}=55 \mathrm{~dB}$ is used. Furthermore, in order to observe how the PSD of the narrowband interference is affecting the five systems, two different PSD values of the narrowband interference, denoted as $\operatorname{PSD}\{\mathrm{NI}\}$, are chosen, namely, $\mathrm{PSD}\{\mathrm{NI}\}=-100$ $\mathrm{dBm} / \mathrm{Hz}$ and $\operatorname{PSD}\{\mathrm{NI}\}=-60 \mathrm{dBm} / \mathrm{Hz}$. Fig. 9 shows the values of the $\mathrm{SNR}_{k}$ for the 32-channel CMTs in the $K=3$ case. The following observations can be made based on these simulations and can also be generalized for other number of subchannels.

1) When comparing the NPR results with those of the PR case, it can be observed that the PR-based CMT is more affected when increasing the PSD $\{\mathrm{NI}\}$ than the four NPR CMTs (the average of the SNR is going down from 55 to $40 \mathrm{~dB}$ ).

2) The GWMT-based CMT has among the four NPR designs the best performance for the considered order of the subchannel filters.

\section{Effects of the Transmission Channel}

When the transmission channel is considered in the GWMT design approach, the optimized values of the weights $A_{i}$ for $i=0,1,2,3$ in the generalized cosine window function and the cut-off frequency of the ideal lowpass filter $\omega_{c}$ for designing a 32-channel CMT are different than the values provided in Table I. For the transmission channel as shown in Fig. 2, the weights $A_{i}$ for $i=0,1,2,3$ and $\omega_{c}$ providing the best performance are given by

$$
A_{0}=0.4444, A_{1}=0.6430, A_{2}=-0.1123, \omega_{c}=0.0217 \text {. }
$$

A comparison between the proposed GWMT-based 32-channel CMT designed by using the parameters, as given by (22) and the CMTs designed by remaining four methods is 


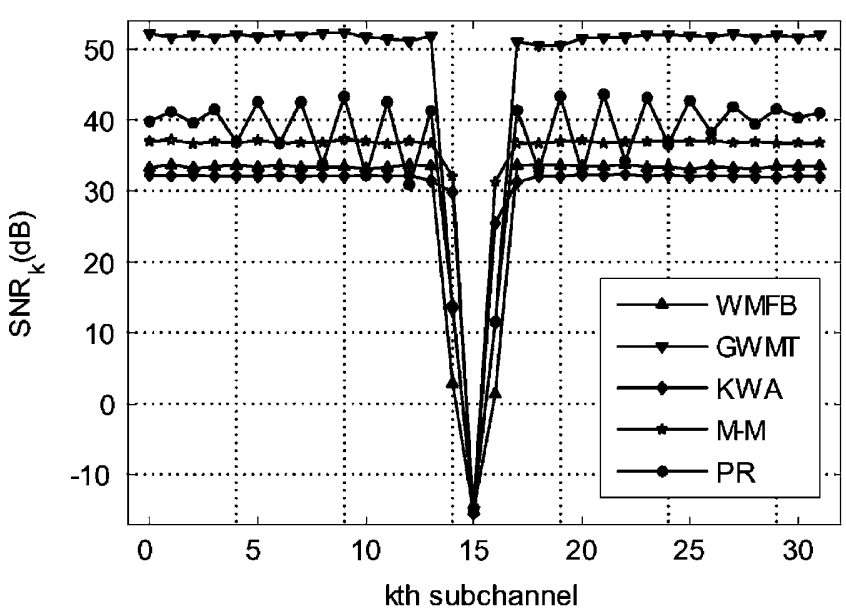

(a)

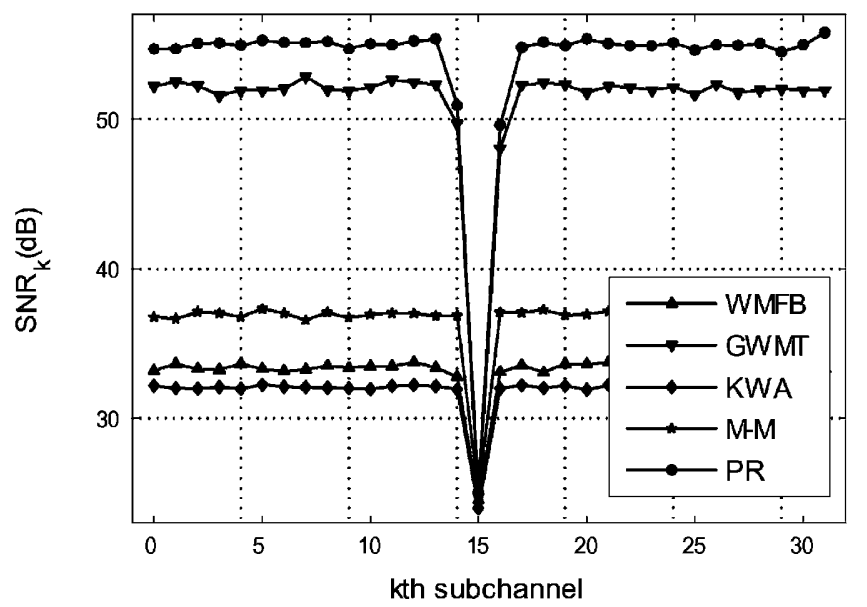

(b)
Fig. 9. Values of the $\mathrm{SNR}_{k}$ for $k=0,1, \ldots, 31$ of the 32-channel CMTs under consideration in the $K=3$ case when a narrowband interference is affecting to the systems. (a) $\operatorname{PSD}\{\mathrm{NI}\}=-100 \mathrm{dBm} / \mathrm{Hz}$. (b) $\operatorname{PSD}\{\mathrm{NI}\}=-60$ $\mathrm{dBm} / \mathrm{Hz}$.

given in Fig. 10. As expected, the SNR depends heavily on the frequency response of the transmission channel, particularly, of its phase response. Furthermore, it is obvious that huge nonlinearities of the transmission channel can not be compensated by designing only one symmetrical window used for designing the prototype filter of the CMTs. Nevertheless, as seen in Fig. 10, the proposed method results in transmultiplexers having better SNRs than transmultiplexers designed by other methods. This indicates that there is an attractive chance to study whether it is possible to improve the overall results by including a proper equalization process in the optimization technique proposed earlier in this contribution.

\section{CONCLUSION}

A simple and efficient design approach for optimizing the prototype filters for NPR CMTs was proposed. In this approach, the trade-off between the interferences for a given transmission channel is controlled during the optimization stage by the proposed objective function. Furthermore, the number of unknowns is only four independently of the order of the subchannel filters

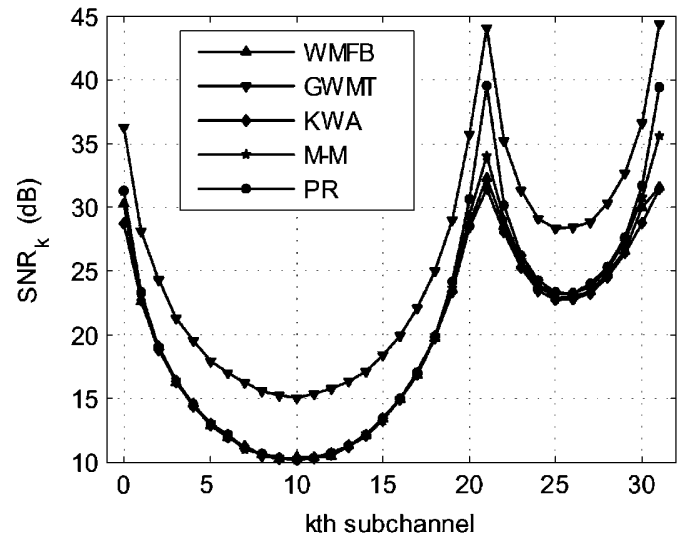

Fig. 10. VALUES of the $\mathrm{SNR}_{k}$ for $k=0,1, \ldots, 31$ of the 32-channel CMTs under consideration in the $K=3$ case over the transmission channel shown in Fig. 2.

and the number of subchannels. This paper also provided tabulated parameters for designing the prototype filters for CMTs without optimization, thereby simplifying the overall design algorithm when the transmission channel is not considered. In this case, it was observed that selecting $\alpha$ to be equal to 0.5 results in CMTs with the highest SI performance, whereas smaller (larger) values of $\alpha$ improve the SISI (SICI). Moreover, the simulation performed in this contributions showed that the highest SNR is achieved by using the proposed design among the other four NPR design approaches under consideration. The design approach of NPR CMTs including the equalization process remains as a topic for future work. Preliminary results indicate that there is a chance to improve the proposed technique by including the equalization process.

\section{REFERENCES}

[1] K. Maxwell, "Asymmetric digital subscriber line: Interim technology for the next forty years," IEEE Trans. Signal Process., vol. 34, no. 10, pp. 100-106, Oct. 1996.

[2] J. Yunho, K. Jiho, L. Seongjoo, Y. Hongil, and K. Jaeseok, "Design and implementation of MIMO-OFDM baseband processor for high-speed wireless LANs," IEEE Trans. Circuits Syst. II, Express Briefs, vol. 54, no. 7, pp. 631-635, Jul. 2007.

[3] J. A. C. Bingham, "Multicarrier modulation for data transmission: An idea whose time has come," IEEE Commun. Mag., vol. 28, pp. 5-14, May 1990.

[4] S. D. Sandberg and M. A. Tzannes, "Overlapped discrete multitone modulation for high speed copper wire communications," IEEE J. Sel. Areas Commun., vol. 13, no. 8, pp. 1571-1585, Dec. 1995.

[5] G. Cherubini, E. Eleftheriou, and S. Olcer, "Filtered multitone modulation for very high-speed digital subscriber lines," IEEE J. Sel. Areas Commun., vol. 20, no. 6, pp. 1016-1028, Jun. 2002.

[6] A. N. Akansu and X. Lin, "A comparative performance evaluation of DMT (OFDM) and DWMT (DSBMT) based DSL communications systems for single and multitone interference," in Proc. IEEE Int. Conf. Acoust., Speech, and Signal Process., Seattle, WA, May 1998, vol. 6, pp. 3269-3273.

[7] F. Cruz-Roldan and M. Monteagudo-Prim, "Efficient implementation of nearly perfect reconstruction FIR cosine-modulated filterbanks," IEEE Trans. Signal Process., vol. 52, no. 9, pp. 2661-2664, Sep. 2004.

[8] A. Viholainen, J. Alhaba, J. Helenius, J. Rinne, and M. Renfors, "Equalization in filter banks based multicarrier systems," in Proc. IEEE Int. Conf. on Electron., Circuits and Syst., Pafos, Cyprus, Sep. 1999, vol. 3, pp. 1467-1470.

[9] H. S. Malvar, "Lapped transforms for efficient transform/subband coding," IEEE Trans. Acoust., Speech, Signal Process., vol. 38, no. 6, pp. 969-978, Jun. 1990. 
[10] R. Bregovic and T. Saramaki, "A systematic technique for designing linear-phase FIR prototype filters for perfect-reconstruction cosine-modulated and modified DFT filterbanks," IEEE Trans. Signal Processing, vol. 53, no. 8, pp. 3193-3201, Aug. 2005.

[11] P. N. Heller, T. Karp, and T. Q. Nguyen, "A general formulation of modulated filter banks," IEEE Trans. Signal Processing, vol. 47, no. 1, pp. 986-1002, Jan. 1999.

[12] A. Viholainen, T. H. Stitz, J. Alhava, T. Ihalainen, and M. Renfors, "Complex modulated critically sampled filter banks based on cosine and sine modulation," in Proc. IEEE Int. Symp. Circuits Syst., Scottsdale, AZ, 2002, vol. 1, pp. 833-836.

[13] T. Karp and N. J. Fliege, "Modified DFT filter banks with perfect reconstruction," IEEE Trans. Circuits Syst. II, Anal. Digital Signal Process., vol. 46, no. 11, pp. 1404-1414, Nov. 1999.

[14] F. J. Harris, "On the use of windows for harmonic analysis with the discrete Fourier transform," Proc. IEEE, vol. 46, no. 1, pp. 51-84, Jan. 1978.

[15] N. J. Fliege, Multirate Digital Signal Process., 2nd ed. New York: Wiley, 1999.

[16] R. D. Koilpillai, T. N. Nguyen, and P. P. Vaidyanathan, "Some results in the theory of crosstalk-free transmultiplexers," IEEE Trans. Signal Process., vol. 39, no. 10, pp. 2174-2183, Oct. 1991.

[17] M. Vetterli, "Perfect transmultiplexers," in Proc. IEEE Int. Conf. on Acoustics, Speech, and Signal Process., Tokyo, Japan, Apr. 1986, vol. 11, pp. 2567-2570.

[18] M. Vetterli, "A theory of multirate filter banks," IEEE Trans. Acoust., Speech, Signal Process., vol. ASSP-35, no. 3, pp. 356-372, Mar. 1987.

[19] P. P. Vaidyanathan, Multirate Systems and Filter Banks. Englewood Cliffs, NJ: Prentice-Hall, 1993.

[20] H. S. Malvar, Signal Processing With Lapped Transforms. Norwood, MA: Artech House, 1992.

[21] P. Martin-Martin, F. Cruz-Roldan, and T. Saramaki, "Optimized transmultiplexers for multirate systems," in Proc. IEEE Int. Symp. on Circuits Syst., Kobe, Japan, May 2005, vol. 2, pp. 1106-1109.

[22] C. D. Creusere and S. K. Mitra, "A simple method for designing highquality prototype filters for M-band pseudo QMF banks," IEEE Trans. Signal Process., vol. 43, no. 4, pp. 1005-1007, Apr. 1995.

[23] Y. P. Lin and P. P. Vaidyanathan, "A Kaiser window approach for the design of prototype filters for cosine modulated filterbanks," IEEE Signal Process. Lett., vol. 5, no. 6, pp. 132-135, Jun. 1998.

[24] H. A. Nuttall, "Some windows with very good sidelobe behavior," IEEE Trans. Acoust., Speech, Signal Process., vol. ASSP-29, no. 2, pp. 84-91, Feb. 1981

[25] K. W. Martin, "Small side-lobe filter design for multitone data-communication application," IEEE Trans. Circuits Syst., vol. 45, pp. 1155-1161, Aug. 1998.

[26] S. Mirabbasi and K. Martin, "Overlapped complex-modulated transmul-tiplexer filters with simplified design and superior stopbands," IEEE Trans. Circuits Syst. II: Anal. Digital Signal Process., vol. 50, no. 8, pp. 456-469, Aug. 2003.

[27] G. B. Dantzig, Linear Programming and Extensions. Princeton, NJ: Princeton Univ. Press, 1963.

[28] J. A. Nelder and R. Mead, "A simplex method for function minimization," Computer J., vol. 7, pp. 308-313, 1965.

[29] T. Coleman, M. A. Branch, and A. Grace, Optimization Toolbox User's Guide, Version 2. Natick, MA: MathWorks, Inc., 1999.

[30] F. Cruz-Roldan, P. Amo-L6pez, S. Maldonado-Bascon, and S. S. Lawson, "An efficient and simple method for designing prototype filters for cosine-modulated pseudo-QMF banks," IEEE Signal Process. Letters, vol. 9, pp. 29-31, Jan. 2002.

[31] Very-High-Speed Digital Subscriber Lines, System Requirements, Draft Standard, T1E1.4/98-043R2, ANSI, May 1998.
Tampere International Center for Signal Processing and in 2004, she was Marie Curie Student Fellow, both in the Department of Signal Processing, Tampere University of Technology. Her research interests are in multirate signal processing and in multicarrier transmission. 
system-level designer of VLSI Solution Oy, Tampere, Finland, specializing in efficient VLSI implementations of both analog and digital signal processing algorithms for various applications. He is also the President of Aragit Oy Ltd., Tampere, Finland, which was founded by four TUT professors and concentrates on spreading worldwide their know-how on information technology to the industry. In 1982, 1985, 1986, 1990, and 1998. He was a Visiting Research Fellow (Professor) with the University of California, Santa Barbara, in 1987 with the California Institute of Technology, Pasadena, and in 2001 with the National University of Singapore. His research interests are in digital signal processing, especially filter and filterbank design, VLSI implementations, and communications applications, as well as approximation and optimization theories. He has written almost 300 international journal and conference articles, various international book chapters, and holds three worldwide used patents.

Dr. Saramäki was a recipient of the 1987 and 2007 IEEE Circuits and Systems Society's Guillemin-Cauer Awards as well as two other best paper awards. In 2004, he was also awarded the honorary membership (Fellow) of the A. S. Popov
Society for Radio-Engineering, Electronics, and Communications (the highest membership grade in the society and the 80th honorary member since 1945) for "great contributions to the development of DSP theory and methods and great contributions to the consolidation of relationships between Russian and Finnish organizations". He is also a founding member of the Median-Free Group International. He was an Associate Editor for the IEEE TRANSACTIONS ON CIRCUITS AND SyStems-II: ANALOG AND Digital Signal PRocessing from 2000 to 2001, and is currently an Associate Editor for Circuits, Systems, and Signal Processing. He has been actively taking part in many duties in the IEEE Circuits and Systems Society's DSP Committee, namely by being a Chairman (2002-2004), a Distinguished Lecturer (2002-2003), a Track or a Co-Track Chair for many ISCAS symposiums (2003-2005). In addition, he has been one of the three chairmen of the annual workshop on Spectral Methods and Multirate Signal Processing (SMMSP), started in 2001. 